\title{
Die Vegetationszonen des nördlichen Eurasiens während der letzten Eiszeit ${ }^{1}$ )
}

\author{
Von B. Frenzel und C. Troll. Mit einer Karte
}

In der vorliegenden Arbeit wird versucht, auf Grund des neuesten russischen Materials die letzteiszeitlichen Vegetationszonen Eurasiens zu rekonstruieren.

Schon früher untersuchten BüDEL (1949) und v. WissmanN (1938) die Landschaftszonen der letzten Eiszeit in Europa und China. Im wesentlichen werden die Forschungsergebnisse der beiden Autoren durch die modernste russische Literatur bestätigt. Daher konnten, nach kleinen Umänderungen, die Karten von v. WissmanN und BüDEL als Ausgangspunkte benutzt werden.

Bevor jedoch die letzteiszeitlichen Vegetationszonen skizziert werden können, muß noch einiges über die Vergletscherung gesagt werden. Unsere Kenntnis über die letzteiszeitliche Vergletscherung West-, Süd- und Mitteleuropas hat keine besonderen Änderungen erfahren. Jedoch gelang es SokoLow (1946), die dem Brandenburger Stadium der Weichseleiszeit entsprechende Eisrandlage auch im europäischen Teil der UdSSR zu verfolgen. Dadurch verändern sich gegenïber BüDEL's Darstellung die Konturen des nordeuropäischen Inlandeises ganz beträchtlich. SokoLow scheint aber, nach den geologischen Verhältnissen zu urteilen, auf der Strecke vom Onega-See zum Weißen Meer das Brandenburger Stadium mit einem späteren verwechselt $\mathrm{zu}$ haben. Deshalb zeichneten wir in diesem Raum die Eisrandlagen neu, indem wir ausgeprägte Sandergebiete und die Verbreitung glazialer Tone als Hinweise auf den Verlauf der ehemaligen Eisrandlage benutzten.

Schon lange war es bekannt, daß der Ural eine Gebirgsvergletscherung getragen hat. Man war sich aber noch nicht darüber im Klaren, wie weit die Vergletscherung nach Süden reichte. So ist es recht interessant, daß KoLokoLow und Ljwow (referiert bei Kотоw 1947) 1945 am Berg Sigalga im Süd-Ural Kare, Tröge usw. oberhalb $1000 \mathrm{~m}$ Höhe entdeckten, also Zeugen einer alpinen Vergletscherung dieses höchsten Gebirgsstockes im Süd-Ural.

Noch immer muß die Ostgrenze der Vergletscherung des Norilsker Plateaus (Quellgebiet der Chatanga) als unbekannt betrachtet werden. DumirRaschko und KAMANIN (1946) gelang es jedoch inzwischen, eine letzteiszeitliche Talvergletscherung im Oberlaufgebiet des Wiljuij nachzuweisen. Sehr wahrscheinlich war aber dieses Vergletscherungsgebiet nur eine Ausnahme in dem sonst unvergletschert gebliebenen N-Teil Mittelsibiriens. Bestenfalls gab es damals noch hier und da festliegende Firnfelder; zu einer nennenswerten Vergletscherung des mittelsibirischen Berglandes ist es aber sicher nicht gekommen.

Lange ging der Streit um die Zahl der Vergletscherungen Mittel- und Ostsibiriens. Auch die Hypothese von Gerassimow \& Markow (1939) über die Zwi-

1) Die Bearbeitung wurde ausgeführt im Rahmen eines größeren Forschungsauftrages der Akademie der Wissenschaften und der Literatur (Sitz Mainz), der der Erforschung des Zustandes der Erdoberfläche während der diluvialen Vereisungen gilt. Eine Serie von größeren Abhandlungen wird demnächst in den Abhandlungen der Akademie, mathem.-naturwiss. Klasse, in Druck gehen. Der vorliegende Bericht mit Karte geht im wesentlichen auf die Auswertung der russischen Literatur durch Herrn B. Frenzel zurück. Sehr wichtige Literatur wurde durch die freundliche Hilfe von Herrn Prof. Dr. H. Gams-Innsbruck und Herrn Prof. Dr. H. Wilhelmy-Kiel zugänglich gemacht. Großen Dank schulden wir auch dem Geographischen Institut der HumboldtUniversität Berlin unter Leitung der Herren Dr. H. Waldbaur und Prof. Dr. F. Haefke, die eine gründliche Einsichtnahme der im Institut befindlichen Literatur gestatteten. 
schenzeitlichkeit der Vergletscherung des genannten Gebietes in Hinblick auf die europäische Chronologie klärte die schwierigen Fragen keineswegs, sondern verursachte nur noch mehr Verwirrung. So ist es sehr zu begrüßen, daß SAKs (1947) den Versuch unternommen hat, auf Grund der Morphologie und der Stratigraphie eine Gliederung des Eiszeitalters in Mittel- und Ostsibirien durchzuführen. Saks stellt fest, daß zwei Eiszeiten und ein Stadium der zweiten Eiszeit noch heute morphologisch nachweisbar sind. Die früheste Eiszeit ist nur stratigraphisch nachweisbar. Die beiden ältesten Vergletscherungen hatten den Charakter eines Inlandeises, die jüngste Eiszeit und ihr Hauptstadium prägten sich als Talvergletscherungen aus, die sich bestenfalls zu unbedeutenden Vorlandvergletscherungen zusammenschlossen. SAKs ist der Meinung (und das wohl mit Recht), daß man die von ihm erkannten Vergletscherungen mit den europäischen zeitlich gleichsetzen kann, und er kommt zu folgendem Schema:

$\begin{array}{lll}\text { Mittel- und Ostsibirien } & \text { Osteuropa } & \text { Mitteleuropa } \\ \text { Älteste Vergletscherung } & \text { Lichwinvereisung } & \text { Elstereiszeit } \\ \text { maximale Vergletscherung } & \text { Dnjeprowsk Vereisung } & \text { Saale-Eiszeit } \\ \text { Syrjanka Vergletscherung } & \text { Moskau Eiszeit } & \text { Warthe-Stadium (?) } \\ \text { Sartan Stadial } & \text { Stadial des Moskau-Glazials } & \text { (Weichsel-Eiszeit?) }\end{array}$

Die Beobachtungen reichen leider noch nicht aus, um die Stellung des Sartanstadials genau feststellen zu können. Es wäre ja denkbar, daß dieses sogenannte Stadial der Waldai-Eiszeit entspräche.

Auf Grund der Angaben von SaKs (1947), Obrutschew (1935), v. Klebelsberg (1949), KLUTE (1928) und Popow (1947) versuchten wir, die Vergletscherung Ostsibiriens darzustellen. Daß die als vergletschert eingezeichneten Berggruppen während der letzten Eiszeit wirklich vereist waren, und daß es sich in der Tat um Talvergletscherungen handelte, ist sicher. Fraglich ist nur das Ausmaß der Vergletscherung. Viel stärker, als hier angenommen wurde, kann die Vereisung nicht gewesen sein, eher ist an eine geringere Eisbedeckung zu denken. Entscheidend ist aber, daß auf jeden Fall die eiszeitliche Schneegrenze in diesem Gebiet beträchtlich höher lag als in der Umgebung, d. h., daß damals ganz ähnliche meteorologische Verhältnisse herrschten wie heute.

Nachdem GoRodkow (1946) festgestellt hatte, daß die botanischen Verhältnisse der Wrangel-Insel darauf hinweisen, daß diese Insel letzteiszeitlich nicht vergletschert gewesen sein kann, machte es SAKs (1947) sehr wahrscheinlich, daß auch die Wrangel-Insel letzteiszeitlich vergletschert war. So beschreibt er Kare, Tröge und Endmoränen, deren Formen zwar durch die junge Solifluktion stark verwischt wurden, die aber doch noch deutliche Zeugen einer beschränkten Karund Talvergletscherung darstellen.

Nach den Forschungen von DAmpserow \& Elisejew war auch Sachalin während der letzten Eiszeit (im Taimyrian) vergletschert. Genaue Angaben über die Art dieser Vergletscherung liegen nicht vor. $\mathrm{Da}$ aber in den Tiefländern beiderseits der Gebirgszüge innerhalb der Sedimente keine Moränen beschrieben wurden, muß man annehmen, daß es sich nur um eine Talvergletscherung gehandelt haben kann. $\mathrm{Zu}$ einer ähnlichen Talvergletscherung kam es auch in der Berggruppe Chamar daban am Baikal See (Сновозснісн 1951) und auf dem Patomplateau an der oberen Lena (Dumitraschko \& Kamanin 1946). Neue Forschungsergebnisse über die letzteiszeitliche Vergletscherung NW-Armeniens teilte Gabrieljan (1951) mit. Er stellte fest, daß die Achotskije, Mokryje und LahwarBerge vergletschert waren. In der vorletzten Eiszeit soll dort keine Vergletscherung stattgefunden haben, da damals die Berge noch nicht hoch genug waren. 
Soweit die Ergänzungen zu den bisherigen Vorstellungen über die letzteiszeitliche Vergletscherung Eurasiens.

BüDEL's Rekonstruktionsversuch der würmeiszeitlichen Vegetationszonen Europas konnte in der letzten Zeit von französischen Forschern in Süd-Frankreich berichtigt werden (mündliche Mitteilung von Prof. BüDEL). Nach diesen neuen Forschungen ergibt es sich, daß der Wald selbst in Süd-Frankreich nördlich der Pyrenäen ganz ausfiel. An die Stelle des Waldes war ein schmaler Keil der Waldund Strauchtundra getreten, der aber auch nicht weit nach Norden reichte, denn dort waren die solifluidalen Vorgänge sehr stark wirksam. Vom Ostrand der Pyrenäen verlief die Wald- und Strauchtundra etwa an der heutigen Südküste Frankreichs entlang zum Süd-Rand der Alpen. Am Westrand der Alpen war keine Strauchtundra vorhanden, und das im Rhônetal von BüDEL angenommene Waldsteppengebiet war wohl ein Mischungsbereich von Tundren- und Steppenpflanzen. Eine ganz ähnliche Vegetation muß wohl auch im Grenzbereich der Steppen und Tundren des pannonischen Beckens angenommen werden.

Nach einer mündlichen Mitteilung von Prof. BüDEL drang in der Gegend von Florenz an Stelle des Mischwaldes mit wärmeliebenden Arten der boreale Nadelwald bis fast an das Mittelmeer vor. Kleinere Veränderungen der Büdelschen Karte mußten auch in Spanien und auf dem Balkan vorgenommen werden.

Gegenüber der Büdelschen Karte ergaben sich aber in Ost-Europa auf Grund der neuen russischen Forschung wesentlich andere Verhältnisse. Bis zu einer Linie, die ungefähr der Eisrandlage des Moskauglazials folgte, stieß von Norden her das Gebiet sehr intensiver Solifluktion vor. In diesem Bereich wälzte sich Fließerde über interglaziale Moore, und Erhebungen wurden durch solifluidale Vorgänge stark abgeflacht und eingeebnet (SokoLow 1946). Diese Zone, die heute im Ural an Hand der fossilen Goletzterrassen ${ }^{2}$ ) zu verfolgen ist (Bотsch \& KrassNow 1951), trat auch noch in der westsibirischen Tiefebene auf, wo die deluvialen ${ }^{3}$ ) Lehme (Sukatschew 1933) bei Demjansk und Tobolsk Zeugen dieser Vorgänge sind. Diese durch die Solifluktion landschaftlich weitgehend bestimmte Zone war aber keineswegs vegetationslos. Zwar fehlte der Baumwuchs, und nur seltene Exemplare von Betula nana und Salix polaris waren dort zu finden. Aber eine arktische Kräuterflora bedeckte das Gelände und nahm nach Süden, entsprechend der schwächer werdenden Solifluktion an Geschlossenheit zu (Solonewitch \& Kortschagin 1934; Gritschuck 1946; Blagowechtschenskij 1946). Etwa 200 bis $300 \mathrm{~km}$ vom Eisrand entfernt, ging diese Landschaft der arktisch-alpinen

2) Als Goletzterrassen (Gebirgsterrassen usw.) bezeichnet man eine eigenartige kleinräumige Stufung der Berghänge. Es handelt sich um nach außen ganz sanft geneigte, im Höchstfall einige Zehner von Metern tiefe und wenige Hundert Meter breite, fast horizontale Verebnungen in der Hanglinie. Diese "Ter assen“ lassen sich nicht zu zusammenhängenden Systemen zusammenfassen. Die Verebnungen sind nur von einer niederen Vegetation bedeckt, und überall herrscht starke Solifluktion. Die Goletzterrassen sind Formen der morphologisch wirksamen Solifluktion.

$\left.{ }^{3}\right)$ In russischen bodenkundlichen Arbeiten treten vielfach uns weitgehend fremde Fachausdrücke auf. Zu ihnen gehören die Bezeichnungen: deluvial und proluvial. Die Termini: alluvial, eluvial und illuvial werden auch bei uns gebraucht.

Von deluvialen Sedimenten spricht man dann, wenn raan nachweisen kann, daß diese Schichten, stark durchfeuchtet, langsam und stetig vom Höheren zum Tieferen geglitten sind. Ihre Schichtfolge ist naürlich vollkommen gestört, aber sie grenzen mit einer scharfen Fläche gegen die nicht bewegten Sedimente. Deluviale Sedimente sind in der Mehrzahl der Fälle unseren Solifluktionslehmen gleichzusetzen, jedoch muß vorher immer geprüft werden, ob diese Parallelisierung tatsächlich anwendbar ist.

Proluviale Sedimente sind die feinen Ablagerungen periodisch oder episodisch abkommender Flüsse in Trockengebieten. Sie weisen immer eine gewisse Schichtung auf und sind nicht so homogen, wie die deluvialen Sedimente. 
Kräuterfluren und der starken Solifluktion in eine Strauchtundra über, in der Ericaceen, Zwergbirken und Zwergweiden recht reichlich vertreten waren. Aber noch immer war die Solifluktion sehr stark. Jedoch schon in der Gegend von Pljoss begann die hochstämmige Birke die Herrschaft zu erringen und deutete so auf Verhältnisse hin, die bei Lichwin besonders deutlich werden. Denn es zeigt sich, daß in diesem Gebiet der Strauchtundra neben vereinzelten Kiefern und Erlen jetzt auch südliche Steppenelemente vorhanden sind, unter denen Chenopodien (bis 5\%) und Artemisia (bis 25\%) besonders auffällig und bezeichnend sind.

Zwischen die arktisch-alpine Kräuterflur im Norden und die -allerdings kühle - Steppe im Süden war demnach ein schmaler Streifen eines eigenartigen Vegetationsgemisches eingeschoben: Tundra und Waldsteppe verzahnten sich und verdrängten die Taiga ganz (GRitschuk 1946; Blagoweschtschenskij 1946).

Es kam also während der letzten Eiszeit in Ost-Europa weder zur Ausbildung einer Waldtundra, wie es Gerassimow \& Markow (1939) annahmen, noch zu einer Waldsteppe (BüDEL 1949), sondern zu einem Gemisch beider Formen. Außerdem reichte diese eigenartige Mischzone viel weiter nach Süden, als es Büdel angenommen hatte, und auch ihre Nordgrenze lag südlicher als die von BüDEL auf Grund der $10,5^{\circ}$ Juli-Isotherme rekonstruierte Nordgrenze der Waldsteppe.

Es wurde eben schon gesagt, daß selbst in der Waldtundra-Waldsteppe die Solifluktion sehr beträchtlich war (Blagoweschtschenskij 1946). Tatsächlich war noch bei Michailow in der Nähe von Rjasan das Klima der Bildung großer Eiskeile günstig (Moskwitis 1940). Das Auftreten dieser Eiskeile und das wiederholte Vorkommen mineralischer Erde in Mooren beweisen, daß die Südgrenze der ewigen Gefrornis während der letzten Eiszeit sehr weit im Süden gelegen hat, wie es Tumel (1946) nachweisen konnte. Nach Tumel verlief die Südgrenze des Gebietes mit Gefrornisinseln von Orenburg über Chwalynsk, Stalingrad, Dnjepropetrowsk nach Tschernowitz.

So ist es nicht verwunderlich, daß die Funde auf eine kühle Steppe hinweisen. Diesen thermischen Charakter beweist einerseits die kälteliebende Fauna. Denn bis zur Krim kamen das Mammut, der Braune Bär, der Polarfuchs, der nördliche Fuchs, das Rentier und der Schneehase vor (Gerassimow \& Markow 1939). Andererseits geben die Reliktvorkommen von Kiefer, Fichte und Lärche auf dem Donezplateau davon Kunde. Und auch Senkow (1938) konnte pollenanalytisch ähnliche Kiefern- und Fichtenhaine in der Ukraine nachweisen. An feuchten Stellen war die Grauerle stark vertreten. Ob es aber zur Ausbildung richtiger Auenwälder gekommen ist, muß noch fraglich bleiben. Wahrscheinlich handelte es sich nur um lose miteinander verbundene Haine, die nach Norden hin immer seltener wurden.

Aus den Bestimmungen der Holzkohlen, die in den Höhlen Ssjuren I und II am Nordabhang des Jailagebirges gefunden wurden, geht hervor, daß auf der nördlichen Krim während der letzten Eiszeit folgende Pflanzen wuchsen: Populus tremula, Sorbus aucuparia, Rhamnus cathartica, Betula sp., Juniperus sp., Salix sp. D. h. es handelte sich um eine Vegetation, die heute $900-1000 \mathrm{~m}$ höher zu finden ist (HAMmermanN 1934).

Faßt man das oben Gesagte zusammen, so ergibt sich folgendes: In einem schmalen Streifen zog sich die nahezu vegetationslose Frostschutt-Tundra am Eisrand entlang und bog an der heutigen Nordküste des Kontinents zum Ural hin um. Weiter im Süden ging die Frostschutt-Tundra in eine kräuterreiche 
Frostschutt-Tundra über, in die einzelne Zwergbirken und Zwergweiden eingesprengt waren.

Die Intensität der solifluidalen Kräfte ließ allmählich nach, und auch das Klima selbst wurde dem Baumwuchs günstiger. Daher traten in der Gegend von Lichwin, Moskau und Pljoss Bäume zahlreicher als bisher auf. Unter ihnen errang die hochstämmige Birke die Herrschaft und, mit Chenopodien und Artemisia vereint, trug sie ein Waldsteppenelement in die Waldtundra hinein. So ist dieser Grenzbereich durch eine eigenartige Mischvegetation gekennzeichnet. Noch immer war die Solifluktion sehr stark. Zwar wurde das Klima nach Süden hin nur langsam milder, sodaß noch bis in die Gegend von Dnjepropetrowsk Gefrornisinseln vorkamen. Aber die Trockenheit nahm empfindlich zu. Daher konnte sich Baumwuchs nur noch in Gestalt weniger Kiefern- und Fichtenhaine an edaphisch günstigen Stellen in der sonst überall herrschenden Steppe halten. Die von Laubbaumhainen eingefaßten Flußniederungen wurden gerne von borealen Waldtieren bei ihrer Wanderung nach Süden benutzt, wo sie in den kühlen Birken-Weiden-Hainen der nördlichen Krim von den Paläolithikern des Aurignacien und des Azilien gejagt wurden. Die ersten nennenswerten echten Wälder stockten wahrscheinlich auf dem Südabhang des Jaila-Gebirges.

Auf dem Ural stieß die Frostschutt-Tundra weit nach Süden vor. Am Konschakowskij Kamjenj liegt beispielsweise die Untergrenze der fossilen Goletzterrassen in $500-600 \mathrm{~m}$ Höhe, und selbst am Jaman tau, also unter $54^{\circ} \mathrm{n}$. Br., verläuft diese Grenze in etwa $600-700 \mathrm{~m}$ Höhe. D. h., bis in $600-700 \mathrm{~m}$ Höhe hinab war am Jaman tau während der letzten Eiszeit kein Baumwuchs möglich, und nur eine arktisch-alpine Kräutervegetation bedeckte die Berghänge. Von dieser Flora wurden vor kurzem noch Relikte auf dem Iremel und auf dem Jaman tau gefunden. Es handelt sich um Dryas octopetala, Androsace Lehmanniana, Crepis chrysantha, Carex hyperborea, Cerastium alpinum und Pedicularis compacta (Botsch \& KRAssNow 1951; Kotow 1947).

Zwar zeigen diese Beobachtungen, daß die Tundra weit nach Süden vorstieß. Andererseits liegen aber auch Funde vor, die Waldvegetation beweisen. Es sind das ein Moorhirsch an der Emba und ein Biber am Irgis (Gerassimow \& Markow 1939), d. h. also, daß der Wald stark nach Süden in heutige Steppengebiete gedrängt war, und daß er dort nur eine sehr geringe Fläche eingenommen haben kann. Es ist aber wichtig, daß diese Wälder an der Emba und am Irgis die westlichsten Ausläufer der letzteiszeitlichen Taiga sind. Bis zum Ural (von Westen her) war die einzige Formation, in der in nennenswerter Menge Bäume wuchsen (Kiefer, Fichte, (Tanne), Birke, Erle, Weide) die Waldsteppe-Waldtundra. Erst vom Ural ab nach Osten trat dann eine lückenhafte (s. u.) Taiga auf, die im Norden von einem schmalen Streifen der so eigenartigen Waldtundra-Waldsteppe begleitet wurde.

Wie war nun die westsibirische Taiga beschaffen?

Tundrenrelikte sind noch heute an den Berghängen des kasakischen Berglandes, die zur Turgaipforte hinabführen, und im kasakischen Bergland selbst zu finden (Gerassimow \& Markow 1939). Sie beweisen, welch geringer Erhebungen es bedurfte, daß die Tundra auch hier im Süden Fuß fassen konnte. Andererseits fand Bүкоw (1933 a und b) Mammuts am Ters akkan und am Ischim in der Kasachischen ASSR. Leider ist das Mammut ja nicht zur Rekonstruktion der Vegetationszonen zu verwenden, da es in verschiedenen pflanzengeographischen Bereichen seine Nahrung suchte. Daher hat der Fund eines Bison priscus am Ischim eine hohe Bedeutung; denn dieses Tier gilt als Vertreter südlicher, 
vorwiegend offener Gebiete (Tugarinow, referiert bei ThIEL 1951). D h. also, daß in der Taiga auch Steppengebiete vorkamen. Und schließlich fand man am Dschilandschik in der Kirgisen-Steppe Holzreste der Eiche und Zapfen von Fichte und Lärche, und bei Nowosibirsk entdeckte man Torf, Holz- und Borkereste von Kiefer, Arve und Birke; auch Schilfrohr, Riedgras und echte Gräser sind dort zahlreich vertreten (Gerassimow \& Markow 1939).

Von einer geschlossenen Taiga, wie wir sie heute kennen, konnte damals also garnicht die Rede sein. Vielmehr handelte es sich um mehr oder weniger dichte Nadelwaldgebiete, die von Steppen- und Tundrenbereichen durchsetzt waren. Diese eigenartige Hainvegetation war sehr licht und ging nach Norden in die Waldtundra-Waldsteppe über. Südlich des kirgisischen Faltenlandes nahm der Anteil wärmeliebender Laubbäume zu, sodaß es an Stelle der Nadelwaldhaine zu Mischwäldern kam. Die Tundrenbereiche verschwanden allmählich, und aus dem Mischwald wurde weiter im Süden die Waldsteppe.

Recht interessante Einzelheiten über diese Taiga-Hain-Vegetation wurden aus der Gegend von Krassnojarsk bekannt. Hier liegen ja die klassischen paläolithischen Fundstätten von Afontowa Gora II, Wojennyj Gorodok und Kokorowskaja (Gromow 1932; Tugarinow 1932; Hammermann 1932, 1934).

In der Terrasse, in der die betreffenden Funde gemacht wurden, kam es damals zur Bildung von Eiskeilen oder Würgeböden, wie aus einer Zeichnung von Gromow (1932) hervorgeht. Das bedeutet, daß das Klima während der letzten Eiszeit auch bei Krassnojarsk noch äußerst rauh war. So ist es durchaus nicht erstaunlich, daß dort eine Avifauna gefunden wurde, die heute auf dem Changai in dem Überschneidungsbereich von alpinen Matten und Hochsteppen lebt. Das weist auf eine letzteiszeitliche Jahrestemperatur von $-6^{\circ} \mathrm{C}$ bei Krassnojarsk hin. Ganz dasselbe ergab auch eine Auszählung der fossilen Säugetiere. Von ihnen waren $25 \%$ für nördliche, offene Landschaften charakteristisch, 8,6\% für südliche, offene Gebiete und 4,4\% für südliche Waldgebiete. Kein einziger Vertreter der nördlichen Wälder ist gefunden worden (nördlich und südlich bezieht sich auf die heutigen Verhältnisse, und zwar nördlich oder südlich von $60^{\circ} \mathrm{n}$. Br.). $\mathrm{Zu}$ diesen sehr bemerkenswerten Funden kommen noch die fossilen Pflanzen: Birke, Lärche, Weide. Sehr wahrscheinlich wuchsen diese Bäume nur in der Nähe der Flüsse.

In der Gegend von Krassnojarsk waren also die physisch-geographischen Verhältnisse während der letzten Eiszeit folgendermaßen beschaffen: Infolge eines trocken-kalten Klimas gab es dort keinen geschlossenen borealen Nadelwald mehr. Die Tundra vereinigte sich mit der Steppe und löste die Taiga in ein Mosaik einzelner Waldgebiete auf, zwischen denen sich eine eigentümliche Tundrensteppe ausdehnte. Tiere vorwiegend nördlicher offener und daneben auch südlicher offener Gebiete bevölkerten die Landschaft und wurden von den $\mathrm{Pa}-$ läolithikern von Afontowa Gora gejagt. Die Winter müssen sehr kalt gewesen sein, und die Frostwirkungen im Boden waren beträchtlich. Diese durch Funde belegten Landschaftsverhältnisse können, wie aus den wenigen, oben zitierten Funden hervorgeht, als charakteristisch fur das westsibirische Taiga-Hain-Gebiet angesehen werden.

Es ist schwierig, über die Vegetation des nördlich der westsibirischen Waldtundra-Waldsteppen-Zone gelegenen Gebietes Aussagen zu machen. Vegetationsfunde liegen nicht vor. Aber in der Chakass-Steppe West-Sibiriens kommen noch heute Pflanzen als Relikte einer ehemals bedeutend größeren Tundraverbreitung vor. KEss (1935) erwähnt außerdem Seen, die die Endstadien fossiler 
Thermokarsterscheinungen sind. Sie liegen östlich von Omsk. Beachtet man ferner, daß noch bei Krảssnojarsk Würgeböden vorkamen, dann nimmt es nicht Wunder, daß die Lösse von Tobolsk, Demjansk und Omsk im Hangenden von deluvialen Lehmen scharf begrenzt werden, und daß westlich Surgut im Gebiet von Konda und Jewre Dünen entstanden (Sukatschew 1933; Edelstein 1936).

In dem ganzen Gebiet nördlich des westsibirischen Waldtundra-Waldsteppegürtels muß also die Solifluktion sehr stark gewesen sein. In den südlichen Bereichen der westsibirischen Tiefebene wird daher damals eine ähnliche Vegetation geherrscht haben, wie zur selben Zeit auch im Nordteil Osteuropas, d. h. eine arktisch-alpine Kräutertundra mit einzelnen Zwergbirken und Zwergweiden. Aber sicher schon an der Konda lockerte sich diese Tundra immer mehr auf und machte der echten Frostschutt-Tundra allmählich Platz.

Zusammenfassend kann also folgendes festgestellt werden: Die echte Frostschutt-Tundra reichte, sich immer mehr auflockernd, nach Süden bis etwa zur Konda. Dann errang eine kräuterreiche Tundra die Herrschaft. Hier kam es noch zu starker Solifluktion, aber es entstanden doch keine Dünen mehr.

Südlich Omsk setzte langsam der Baumwuchs ein, und ähnlich wie in Ost€uropa durchdrangen sich Waldtundra und Waldsteppe. Allmählich wurden die Haine dichter, und Holzarten des borealen Nadelwaldes traten in ihnen auf. Es ist das das Gebiet der Taiga-Haine und der kalten Steppen, in denen sich vorwiegend kälteliebende Tiere offener Landstriche aufhielten. Zum großen Teil stockte auch diese Mischvegetation auf der ewigen Gefrornis, deren Südgrenze vom Tarbagatai zum Ost-West gerichteten Lauf des Ischim und von dort nach Orenburg verlief (Tumel 1946). Südlich des kirgisischen Faltenlandes fielen die nördlichen Pflanzenarten immer mehr aus, und Eichen und andere wärmeliebende Holzarten traten in den Wäldern auf, deren Südgrenze gegenüber der Waldsteppe heute noch unklar ist.

Aus dem mittelsibirischen Raum sind bisher nur sehr wenige Funde bekannt geworden, die etwas über die letzteiszeitlichen Vegetationsverhältnisse aussagen können.

Aus der weiten Verbreitung zahlreicher Pflanzen- und Tierarten schließen Dumitraschkow \& Kamanin (1946), daß sich die Vegetationsverhältnisse Mittelsibiriens während der Eiszeiten gegenüber den heutigen nicht wesentlich geändert haben können. Vielfach sind ja aus der Verbreitung von Pflanzen- und Tierarten abgeleitete Beweise nicht direkt zwingend. Daher ist es ganz besonders wichtig, daß die beiden Forscher mit Hilfe der Pollenanalyse feststellten, daß im Baikalgebiet und im Lenabergland während des ganzen Quartärs dieselben Nadelwälder wie heute stockten.

Ein weiterer Hinweis auf die ehemaligen Wälder ist auch die Verbreitung der Alasse, d.h. der Grassteppengebiete in der Nähe von Seen innerhalb der Taiga. Das Hauptgebiet der Alasse ist die Umgebung der mittleren Lena, des unteren Wiljuij und des unteren Aldan. Hier bedecken lößähnliche Lehme, in denen in 2 Meter Tiefe die ewige Gefrornis auftritt, Wasserscheiden und Flußterrassen. Von den Lößlehmgebieten sagte schon Grigorjew (referiert bei Gerassimow \& Markow 1939): „So breiteten sich in dieser Senke südlich der Eisrandlage der Gletscher des Werchojansker Gebirges weite, unbewegliche Firnfelder aus. Diese wurden sowohl durch das im Sommer anfallende Schmelzwasser, als auch durch die Winde mit Staub und Schlamm zugeschüttet. Das fand aber nicht nur hier im Norden statt, sondern im verstärkten Maße sicher auch im Süden, wo ja damals Polarwüsten oder Halbwüsten mit einer starken Verwitterung ge- 
legen waren." Die eigenartige lokale Verbreitung dieser Alasse legt den Gedanken nahe, daß um dieses kalt-trockene Gebiet herum eine dichtere Vegetation stockte. Sehr wahrscheinlich war es der Wald, der eine Lößbildung auf einer größeren Fläche unmöglich machte.

Für diese Annahme spricht die außerordentlich große Ähnlichkeit im Verlauf der letzteiszeitlichen Isochionen ${ }^{4}$ ) mit dem der heutigen. Denn diese Tatsache besagt, daß das fossile meteorologische Regime dem heutigen stark glich. In Mittelsibirien war es also wohl kälter als heute, aber die Kontinentalität war genau so stark, wenn nicht sogar stärker als heute. Daher stieß in der letzten Eiszeit der Wald in Mittelsibirien sehr wahrscheinlich unverhältnismäßig mehr nach Norden vor, als weiter im Westen in den maritimeren Bereichen. Die gleichen Verhältnisse finden sich in der heutigen Vegetation. Denn die einander entsprechenden Vegetationszonen liegen im allgemeinen in Mittelsibirien um $2-5^{\circ}$ nördlicher als in West-Sibirien.

$\mathrm{Daß}$ in der Nähe der Alasse tatsächlich während der letzten Eiszeit Wald stockte, nehmen auch Gerassimow \& Markow (1939) an: „Das ist der Landschaftstyp, in dem einst und jetzt die Verlössung in Jakutien stattfand. Die alles beherrschende Taiga auf den krümeligen Karbonatböden; die Grassteppeninseln (Alasse); die teils bewaldeten, alten Flußterrassen; die Wiesen und die Weidengehölze in den Flußauen: Das sind die Elemente des Landschaftstyps, der doch so sehr von den waldlosen Steppen und Wüsten des Südens verschieden ist."

Aber das Bild der letzteiszeitlichen mittelsibirischen Taiga wäre unvollständig, wenn man nicht noch die Funde bei Markowo an der Lena, etwa unter $57^{\circ}$ n. Br., berücksichtigte (DumitraschKo \& Kamanin 1946). Hier kommen in allen Terrassen, bis hinauf zur fünften, folgende Pollenspektra vor: Nichtbaumpollen $72-100 \%$. Von diesen sind $98-100 \%$ Kräuter, bis $49 \%$ Artemisia und nur ganz vereinzelt Gramineen. Daneben kommen aber auch die Pollen der typischen Taiga in beweisender Menge vor. Die 5. Lenaterrasse wird teils als in die vorletzte Eiszeit gehörend angesehen, teils als jünger erachtet. Daraus ersieht man, wie unsicher die Datierung der Sedimente in den dortigen Gebieten noch ist. Aber aus der Tatsache, daß diese Pollenspektren in allen Terrassen gefunden werden, geht doch hervor, daß es sich um eine Gesetzmäßigkeit in der Vegetationsverteilung handelt, die mindestens schon seit der letzten Eiszeit bestanden haben muß.

Neben den zahlreich vertretenen Nichtbaumpollen fällt auch die große Menge an Sporen auf (28-56\%). So kommen Dumitraschкo \& Kamanin zu dem Schluß, daß letzteiszeitlich die Taiga am Mittel- und Oberlauf der Lena an edaphisch und kleinklimatologisch günstigen Stellen von einer Kräuter- und Wermutsteppe durchsetzt war, in der auch häufig Moore auftraten. „Daher ist es durchaus möglich, daß während der Eiszeit eine Sumpfsteppe vorhanden war."

Nach all dem Gesagten muß man sich also die Taiga der letzten Eiszeit in Mittelsibirien ganz ähnlich der heutigen vorstellen: Weite Nadelwälder aus Lärche, Fichte, Arve und im Süden auch Kiefer waren zweifellos das beherrschende Element; aber in diese dunklen Wälder waren häufig an edaphisch und kleinklimatologisch günstigen Stellen Steppen- und Sumpfgebiete eingeschaltet,

4) Leider werden unter dem Terminus „Isochione“ verschiedene Begriffe verstanden, nämlich die Linie, die die Punkte gleicher Schneegrenzhöhe miteinander verbindet und die Linie gleicher Zahl von Tagen mit Schneefall. Im folgenden gebrauchen wir mit Bübel und Brusch die Bezeichnung „Isochione“ für die Linie gleicher Schneegrenzhöhe. 
die sich besonders an der mittleren Lena zu weiten, offenen Steppen oder Waldsteppen zusammenschlossen.

Die Nordgrenze der mittelsibirischen Taiga zu bestimmen, ist recht schwer; denn es liegen keine pollenanalytische Beweise oder sonstige Fossilfunde vor. Leider fällt auch die Flora des „Mammutkomplexes“ aus, da erstens das Mammut in verschiedenen Vegetationszonen lebte, und da fast alle Mammutkadaver unter stratigraphisch unklaren Verhältnissen gefunden wurden. So ist man bei der Rekonstruktion der Nordgrenze der Taiga während der letzten Eiszeit nur auf indirekte Schlüsse angewiesen.

Zwei Anhaltspunkte sind vorhanden: Das ist erstens die Vergletscherung des Norilsker Plateaus. Es ist kaum denkbar, daß der Wald von Süden her bis nahe an den Eisrand heranreichte, oder daß gar das Norilsker Plateau von Wald eingeschlossen war. Der andere Anhaltspunkt ist die erst vor kurzem entdeckte Vergletscherung im Quellgebiet des Wiljuij um den See Sjurjundä (Dumitraschкo \& Kamanin 1946). Dieses Hochland ist ja nur ein Teil des ungefähr Ost-West streichenden, gesamten Wiljuij-Gebirges von ungefähr gleicher Höhe. Daher ist es unwahrscheinlich, daß die Taiga über den Wiljuij noch sehr weit nach Norden vorstieß. Vermutlich verlief also die Nordgrenze der sich nach Norden in eine Waldtundra auflösenden Taiga noch südlich des oben genannten Gebirgszuges und vereinigte sich, ungefähr am Oberlauf der Unteren Tunguska nach SW umbiegend, noch südlich des Pijaja-Gebirges mit dem Taiga-Steppengebiet von Krassnojarsk, das ja nur eine extrem aufgelockerte Variante der mittelsibirischen Taiga darstellte.

Bisher wurden die letzteiszeitlichen Vegetationsverhältnisse NO-Sibiriens mit Hilfe der Flora des "Mammutkomplexes" rekonstruiert. Auf Grund dieser Beobachtungen glaubten viele russische Forscher schließen zu müssen, daß selbst während der letzten Eiszeit Nadel- und Laubwald bis fast an die Gletscher der verschiedenen Gebirge herangereicht habe (Letztes Sammelreferat bei LeNDL 1948). Es wurde aber schon oben gesagt, daß der „Mammutkomplex“ pflanzengeographisch nicht beweisend ist. Auch rein theoretisch ist eine Waldvegetation während der letzten Eiszeit in NO-Sibirien nicht anzunehmen.

Die Richtigkeit der Vermutung erweist die Arbeit von Neustadt \& Tuulina (1936). Eigenartigerweise scheint diese Arbeit vollkommen in Vergessenheit geraten zu sein. Das Untersuchungsgebiet der beiden Forscher war das Anadyrbecken. Am Mittellauf des Main, eines rechten Nebenflusses des Anadyr, brachten sie eine Bohrung nieder, die zwei eiszeitliche und eine zwischeneiszeitliche Schichtfolge durchsank. Der letzteiszeitliche Horizont ist durch eine Tundrenvegetation gekennzeichnet, in der sehr wenige Holzgewächse vorhanden waren. Unter ihnen herrschten die Birke und die Weide vollkommen (Betula sp. bis 95\%, Salix sp. bis $8 \%$ ). Die Vegetation muß also der von Totjma im europäischen Teil der UdSSR (Solonewitsch \& Kortschagin 1934) sehr ähnlich gewesen sein Subkow (1931) berichtet von spät- oder postglazialen Sedimenten desselben Gebietes. Aus seiner Fundbeschreibung geht hervor, daß hier große Massen feinen Schlammes abgelagert wurden, die den von TABER (1943) beschriebenen sehr ähnlich zu sein scheinen. Wahrscheinlich besteht dieser Schlamm des Anadyrgebietes aus den bei der intensiven Solifluktion anfallenden feinsten Bodenpartikeln. Nach Subkow und Neustadt-Tuliana war aber im Spät- oder Postglazial die pflanzliche Bodenbedeckung dort recht dicht, sodaß es fraglich ist, ob die während dieser Zeit stattfindende Solifluktion so große Schlamm-Massen liefern konnte. Es ist wahrscheinlicher, daß es sich um letzteiszeitliches Solifluktionsmaterial handelt, das durch die im Spätglazial einsetzende Degradation der Gefrornis mobil ge- 
macht, im Spät- und Postglazial in die Täler befördert wurde und die vorhandenen Talmulden stark auffüllte.

Sollte sich diese Annahme bewahrheiten, dann wäre die Parallele mit den nordosteuropäischen Verhältnissen auch in den nordostasiatischen Tiefländern vollständig: Eine lockere arktische Tundra, in der nur sehr wenige Weiden und Birken stockten, bedeckte das Gelände, in dem die Solifluktion fast der einzige, die Erdoberfläche gestaltende Faktor war.

Südlich des Aldan griff die Taiga wahrscheinlich über die Lena hinüber und bedeckte das ganze Gebiet bis zu den Waldsteppen und Steppen der Mandschurei. Jedoch sind uns zum Beweis dieser Annahme bisher keine Funde bekannt.

Betrachtet man noch einmal das oben dargelegte, dann kommt man zu folgenden Ergebnissen:

1.) In Osteuropa verdrängte eine eigenartige Mischvegetation aus Waldtundra und Waldsteppe die Taiga vollständig.

2.) Die Ähnlichkeit im Verlauf der letzteiszeitlichen und der rezenten Isochionen legt es nahe, daß während der norddeutschen Weichseleiszeit fast dasselbe meteorologische Regime wie heute herrschte. Diese Tatsache besagt aber, daß der Baumwuchs in Osteuropa erstens durch das maritim-arktische Klima im Norden und zweitens durch das aride Klima im Süden unmöglich gemacht wurde, und nur in dem schmalen Streifen zwischen den beiden Klimaten kam es zur Ausbildung einer baumreichen Vegetation.

3.) Die Zunahme der Kontinentalität ermöglichte einen, allerdings lockeren, Waldwuchs in demselben klimatischen Übergangsgebiet in Westsibiren.

4.) Erst in Mittelsibirien stockte unter einem äußerst kontinentalen Klima die echte Taiga, die von Mooren und Steppengebieten durchsetzt war.

5.) Im gebirgigen NO-Sibirien bedeckte eine arktisch-alpine, lockere Tundra das Gelände, eine Folge des äußerst rauhen, kalten Klimas.

Es ist schwer, schon heute etwas über die Steppen und Wüsten Mittel- und Zentralasiens während der letzten Eiszeit aussagen zu wollen. So kann nur auf einzelne Funde hingewiesen werden und auf die Konsequenzen, die in vegetationskundlicher Hinsicht aus ihnen gezogen werden müssen.

Aus den Berichten von Schultz (1928), v. Klebelsberg (1922) und MachatscHeк (1912) geht eindeutig hervor, daß in den das Tiefland von Turan umgebenden mittelasiatischen Gebirgen während der letzten Eiszeit sehr mächtige und weit ausgedehnte Fanglomerate entstanden. Das heißt aber, daß selbst an dem Austritt der zahlreichen periodischen oder episodischen Flüsse aus den Gebirgen in die Ebene kaum eine dichte, geschlossene Vegetation hat gedeihen können. Wahrscheinlich hatten wir es dort mit einer Steppe zu tun. Auffallend ist aber doch, daß die auf diesen Schuttkegeln liegenden Löße meist ein spätglaziales Alter haben. Das bedeutet, daß die Vegetation so dicht war, daß es zu keiner nennenswerten Lößbildung gekommen sein kann. Anders war es in einzelnen, besonders trockenen Becken innerhalb der Gebirge. Hier kam es, wie es MAснатsCHEK (1912) für das Tschatkaltal im Tienschan sehr wahrscheinlich machen konnte, während des Höchststandes der letzten Eiszeit vielfach zur Lößbildung. In diesen Becken muß die Vegetation also etwas lockerer gewesen sein.

Leider liegen keine Funde vor, die das Vorhandensein von Wäldern zwischen der Steppe am Fuß der Gebirge und der Hochgebirgstundra bewiesen. Jedoch ist es kaum anzunehmen, daß diese Wälder in der letzten Eiszeit verschwunden sein sollten. An den in, die Steppe vorstoßenden Flüssen stockte ein Laubmischwald, 
der auch sehr wahrscheinlich die ziemlich vergrößerten Binnenseen umgab: Aral-See und Balchasch-See. Fedonowitsch (1946) konnte nachweisen, daß ähnliche Wälder auch den Kaspi-See umsäumten. Allerdings werden die Saumwälder nicht besonders dicht gewesen sein. Denn FEDORowitsch fand in diesem Streifen Knochen von Wildschwein, Hase und Kamel. Sehr wahrscheinlich durchdrangen sich auch hier Wald und Steppe. Das wird wohl ganz besonders für die Gebiete gegolten haben, in denen der Kaspi-See keinen Zufluß hatte, also etwa in der Umgebung des Kara-bugas.

Zwar liegen bis jetzt noch keine Funde vor, die Aussagen über die letzteiszeitliche Vegetationsdecke Tibets zulassen. Es ist aber doch nach allgemein klimatologischen Überlegungen sehr wahrscheinlich, daß sich damals dort die Frostschutt-Tundra ausdehnte.

Zwischen dieser Frostschutt-Tundra und der Wüste Takla-makan vermittelte, wie auch heute, die Steppe. Ihre Untergrenze reichte aber tiefer hinab (Huntington 1907). Huntington stellte fest, daß das Gebiet rezenter Lößbildung im Kun-lun weiter oben in den Bergen liege, als der Bereich fossiler Löße, die heute, frei von jeder Vegetation, durch die typische Badlanderosion abgetragen werden. Da zur Lößbildung aber immer eine gewisse Vegetationsbedeckung nötig ist, mußte damals die Steppe weiter hinab gereicht haben als heute. Es ist das eben das Ergebnis stärkerer Bodenfeuchte und herabgesetzter Verdunstung, die natürlich auch die Höhe des Seespiegelstandes des Lob-nor beeinflußten. Ob aber der Lob-nor tatsächlich so groß gewesen ist, wie das NoRIN (1932) annahm, ist doch sehr fraglich. Vermutlich sind in Norin's Darstellung mehrere verschieden alte Seebecken zu einem einzigen zusammengefaßt. Das gleiche gilt wohl auch für den großen See in NW-Tibet, dessen Existenz während der letzten Eiszeit von Trinkler (1922) behauptet wurde. De Terra querte mehrmals dieselbe Gegend und berichtete nie von Strandterrassen, die auf einen so großen See hätten schließen lassen können. So wird wohl TrinkLER mehrere Seen miteinander vereinigt haben. Das ist um so leichter möglich, als die meisten tibetischen Seen viele Terrassen haben; jedoch konnte bis heute noch keine Altersdatierung und Synchronisierung der einzelnen Seeterrassen durchgeführt werden.

Vom S-Rande des Tien-Schan fehlen vollkommen Funde fossiler Vegetation oder fossiler Faunen. Jedoch scheint die weite Verbreitung von fast ausschließlich Schwemmlössen (Machatschex 1914) am Fuß dieses Gebirges darauf hinzudeuten, daß reichlich Schmelzwässer von den Gletschern zum Tarimbecken flossen. Wahrscheinlich konnte unter diesen Umständen dort auch eine reichere Steppenvegetation als heute gedeihen.

In der zentralen Senke von Tannu-Tuwa, nämlich im Tolatal unterhalb Urga, beobachtete Granö (referiert bei Оввuтschew 1930/31) Grusschotter, die aus der vorletzten Eiszeit stammen sollen. Über diesen Schottern liegt Löß, der zwei Bodenprofile enthält. Zwar fehlt jede nähere zeitliche Datierung. Aber es ist doch $z u$ vermuten, daß ein Bodenhorizont aus der letzten Eiszeit stammt. Eine einigermaßen dichte Gras-, vielleicht auch Buschvegetation wäre dann dort sehr wahrscheinlich.

v. Wissmann widmete, wie eingangs erwähnt wurde, den letzteiszeitlichen Vegetationszonen Chinas eine eigene Arbeit. Daher können wir uns hier auf eine Zusammenfassung beschränken. Durch die Forschungen von Barbour (1933) wurde klar, daß der Malan-Löß N-Chinas zum Teil noch letzteiszeitlich ist. Dieser Löß reicht aber nur bis an den Anfang der letzten Eiszeit zurück; denn es befindet sich in dem Malanlöß kein fossiler Bodenhorizont, der ein höheres Alter der unteren Schichten beweisen könnte. Gleichalt mit den unteren Schichten des 
Malanlösses sind der Hsia-schu-Lehm und der Nanking-Lehm, die man nach v. WissmanN's (1938) Forschungen als sicher letzteiszeitlich ansprechen kann.

Die Lößsteppe dieser Zeit stand, mindestens im Norden, unter dem Einfluß eines kalten, trockenen Klimas. Das beweisen die in N-China gefundenen Fossilien: Mammut und Wollhaariges Nashorn (v. KLebelsberg 1949). An der S-Grenze des Lößsteppengebietes wuchsen aber wärmeliebende Bäume. wie z. B. Celtis, die sich wohl zu Waldsteppen zusammenschlossen und in die Mischwälder des Südchinesischen Berglandes überleiteten.

Fassen wir nochmals das oben gesagte zusammen: Ein Vergleich des Verlaufes der rezenten und der letzteiszeitlichen Isochionen ergibt, daß das meteorologische Regime während der letzten Eiszeit dem heutigen sehr ähnlich war. Die Depression der Schneegrenze läßt sich zwanglos durch eine Herabsetzung der allgemeinen Jahrestemperatur erklären, bei der die Verminderung der Sommertemperatur besonders wichtig, ja ausschlaggebend war. Keinesfalls ist für die Verschiebung der Isochionen eine Verstärkung der Niederschläge verantwortlich zu machen. Das beweisen auch die letzteiszeitlichen Vegetationsverhältnisse. Denn in ihnen spiegeln sich ja die heutigen Vegetationszonen wieder, nur daß eben jegliche Vegetation unter einer verminderten Wärmezufuhr zu leiden hatte.

Die Verstärkung der polaren Kaltluft machte sich besonders in dem stark periodischen Klima Ostasiens bemerkbar, wo durch die Südverlagerung der Zyklonenzugstraßen Nordchina weniger Feuchtigkeit zugeführt wurde als heute, sodaß sich das Lößsteppengebiet nach Süden ausdehnen konnte. Diese Tatsache der Vergrößerung der Lößsteppen Chinas weist aber auch darauf hin, daß sich die Abkühlung des Sommers besonders in einer Verlängerung des Winters ausprägte. Denn nur dieses Ereignis konnte das Nordwest-Monsun-System so stärken, daß das der Lößbildung so günstige trocken-kalte Klima eine maximale Dauer erreichte.

Die Eiszeit war also eine Zeit herabgesetzter Jahres-, besonders aber verminderter Sommertemperatur. Das damals arbeitende und bis heute noch funktionierende meteorologische Regime differenzierte die Auswirkung der Abkühlung in den verschiedenen Gebieten Eurasiens und führte vielfach zu recht eigenartigen Vegetationsverhältnissen.

\section{Literaturverzeichnis}

Barbour, G. B.: Pleistocene History of the Huangho.-Bull. Geol. Soc. America 44, S. 1143 ff. 1933.

BlagoweschtschenskiJ, G. A.: Waldgeschichte des ehemals vereisten Gebietes im europäischen Teil der UdSSR im Zusammenhang mit den Klimaschwankungen des Quartärs. - Arb. geogr. Inst. Akad. Wiss. UdSSR 37, S. 267-292. MoskauLeningrad 1946.

Botsch, S. G. \& Krassnow, J. J.: Der Prozeß der Goletz-Einebnung und die Bildung der Gebirgsterrassen. - Priroda 1951, Nr. 5, S. 25-35.

BüDEL, J.: Die räumliche und zeitliche Gliederung des Eiszeitklimas. - Naturwiss. 1949, S. $105-112,133-140$.

Byкow, G. E.: Quartäre Sedimente der Kreise Atbasar und Jesilsk der Kasakischen ASSR.-Nachr. staatl. geogr. Ges. UdSSR 65, S. 241-249. 1933. - Relief und Seebecken des Flusses Ters akkan in Kasachstan. - Ebendort 65, S. 408 bis 423.1933.

Сновоsснісн, P. P.: Chamar-daban. - Priroda 1951, Nr. 3, S. 55-58.

Dampserow, D. J. \& Elisejew, D. N.: On the Quaternary Deposits of the North Sakhalin, in Connection with the Problem of Quaternary Terraces. - Transact. 2. Internat. Conf. Assoc. Study Quaternary Period in Europe. Fasc. III, Seite 145-153. 
Dumitraschкo, N. W. \& Kamanin, L. G.: Paläogeographie Mittelsibiriens und des Baikalgebietes. - Arb. geograph. Instit. Akad. Wiss. UdSSR 37, S. 132-151. MoskauLeningrad 1946.

Edelstern, Ja. S.: Geomorphologischer Abriß der Westsibirischen Tiefebene. - Arb. Inst. phys. Geogr. Akad. Wiss. UdSSR. Heft 20, Moskau-Leningrad 1936.

Fedorowitsch, B. A.: Paläogeographische Probleme der mittelasiatischen Ebenen. Arb. geogr. Inst. Akad. Wiss. UdSSR 37, S. 152-173. Moskau-Leningrad 1946.

Gabrieljan, G. K.: Spuren einer ehemaligen Vergletscherung in NW-Armenien. Priroda 1951, Nr. 4, S. 57-59.

Gerassimow, I. P. \& Markow, K. K.: Die Eiszeit auf dem Territorium der UdSSR. Arb. geogr. Inst. Akad. Wiss. UdSSR 33, Moskau-Leningrad 1939.

Gorodkow, B. N.: Quartäre Geschichte der Vegetation des arktischen Beringmeergebietes. - Ebendort 37, S. 328-329. 1946.

Gritschuk, W. P.: Zur Vegetationsgeschichte des europäischen Teiles der UdSSR im Quartär. - Ebendort 37, S. 249-266. 1946.

Gromow,W. J.: Geologie und Fauna der paläolithischen Fundstätte Afontowa Gora II. Paläolithischer Fundplatz Afontowa Gora II. - Arb. Komm. Erforsch. d. Quartärs. Akad. Wiss. UdSSR, Leningrad 1932, Seite 145-184.

Hammermann, A. G.: Kohlenreste aus paläolithischen Funden bei Afontowa Gora II. Ebendort S. 131-135 (deutsch). 1932. - Ergebnisse der Untersuchung der quartären Flora auf Grund der Holzkohle. - Arbeiten 2. internat. Konf. Ges. Erforsch. Quartärs in Europa. Heft 5, S. 66-74. Leningrad-Moskau-Nowosibirsk 1934.

Huntington, E.: Some Characteristics of the Glacial Period in Nonglaciated Regions. Bull. Geol. Soc. America 18, S. 351-388. 1907.

KEsS, A. S.: Über die Entstehung der Becken der westsibrischen Tiefebene. - Arb. Inst. phys. Geogr. Akad. Wiss. UdSSR, Heft 15, S. 62-118, Moskau-Leningrad 1935.

KLebelsberg, R. v.: Beiträge zur Geologie West-Turkestans. Innsbruck, 1922. - Handbuch der Gletscherkunde und Glazialgeologie, Wien 1949.

Klute, F.: Die Bedeutung der Depression der eiszeitlichen Schneegrenze für eiszeitliche Probleme. - Z. f. Gletscherkunde 16, S. 70-93. 1928.

Koтow, M. J.: Die Hochgebirgsflora- und Hochgebirgsvegetation des südlichen Urals. Sowjetskaja Botanika 15, S. 145-146. 1947.

LENDL, E.: Neuere gletscherkundliche Forschungen im nordöstlichen Sibirien. - Mitt. geogr. Ges. Wien 90, S. 112-114. 1948.

Machatschek, F.: Der westliche Tien schan. - Pet. Mitt. Eng.-Heft 178, 1913.

Moskwitin, A. J.: Eiskeile, keilartige Spalten und ihre stratigraphische Bedeutung. N. Jb. f. Min. etc. 1940 . III, S. 948.

Neustadt, M. J. \& TJulina, L. N.: Zur Geschichte der quartären und postquartären Flora im Gebiet des Flusses Main, eines rechten Nebenflusses des Anadyr. - Arb. Arktis-Inst. 40, S. 259-280. Leningrad 1936.

Norin, E.: Quaternary Climatic Changes within the Tarim Basin. - Geogr. Review 22, S. 591-598. 1932.

Obrutschew, W. A.: Die Verbreitung der Eiszeitspuren in Nord- und Zentralasien. Geol. Rundschau 21/22, 1930/31.

Obrutschew, S. W. \& SAlischtschew, K. A.: The Mountain Systems of North-Eastern Asia. - Geogr. Review 25, 1935.

Popow, Ju. N.: Rezente Gletscher im Indigirka-Einzugsbereich. - Priroda 1947, S. 41-42.

SAks, W. N.: Galb es ehemals Gletscher auf der Wrangel-Insel? - Priroda 1947, H. 12, S. 50-51. - Die quartäre Vereisung Nord-Sibiriens. - Ebendort H. 4, S. $16-25.1947$.

Schultz, A.: Morphologische Beobachtungen in der östlichen Kara kum-Wüste. - Z. f. Geomorphologie 1928.

Serkow, D. K.: Die Moore der Ukrainischen Sowjet-Republik. Floristik und Stratigraphie. - Akad. Wiss. Ukrain. Sowjet-Rep. Kiew 1938.

SoкоLow, N. N.: Über den Verlauf der Eisrandlagen im europäischen Teil der UdSSR. Arb. geogr. Inst. Akad. Wiss. UdSSR 3\%, S. 83-109. Moskau-Leningrad 1946.

Solonewitsch, K. J. \& Kortschagin, A. A.: Über eine fossile arktische Flora bei der Stadt Totjma. - Sowjetskaja Botanika 1934, S. 149-159.

Subkow, A. J.: Über den Charakter einiger quartärer Sedimente NO-Asiens. - Nachr. Akad. Wiss. UdSSR, math.-nat. Kl., 77. Reihe, Nr. 9. Leningrad 1931.

SukAtschew, W. N.: Erforschung der quartären Sedimente im unteren Irtyschgebiet. Expeditionen der Allunions-Akademie der Wissenschaften im Jahre 1932. Akad. Wiss. UdSSR. Leningrad 1953, S. 166-178. 
TABER, St.: Perennially Frozen Ground in Alaska: Its Origin and History. - Bull. Geol. Soc. America 54, S. 1433-1548. 1943.

ThIEL, E.: Die Eiszeit in Sibirien. - Endkunde 5, S. 16-35. 1951.

TrunkLer, E.: Tibet, sein geographisches Bild und seine Stellung im asiatischen Kontinent. - Mitt. geogr. Ges. München 15, 1922.

Tugarinow, A. Ja.: Zur Charakteristik der quartären Avifauna Sibiriens. Paläolithischer Fundplatz Afontowa Gora II. - Arb. Komm. Erforsch. Quartärs. Akad. Wiss. UdSSR Leningrad 1932, S. 115-129.

TumEL, W. F.: Zur Geschichte der ewigen Gefrornis in der UdSSR. - Arb. geogr. Inst. Akad. Wiss. UdSSR 37, S. 124-132. Moskau-Leningrad 1946.

Wissmann, H. v.: Über Lößbildung und Würmeiszeit in China. - Geogr. Z. 44, S. 201-220. 1938.

\section{An hang}

Eiszeitgliederungen im eurasiatischen $\mathrm{R}$ a $\mathrm{m}$.

Mitteleuropa

Polen

europäischer Teil der UdSSR

(n. WOLDSTEDT)

Weichsel

(Warthe)

Saale

Elster

?

Kaspi-See

(n. Gerassimow \& Mrakow)

Chawalynsk

?

Chasar

Baku

?

China

(n. v. WISSMANN)

Tali

?

Luschan

Taku

Poyang (n. Permik, Piech, TscharNOTZKIJ)

Warschau II

?

Warschau I

Krakau

Jaroslawl (?) (n. Gerassimow \& Markow, SoKoLow)

Waldai

(Moskau)

Dnjeprowsk

Lichwin

?

Mittel- und Ostsibirien

Altai

(n. SAKs)

(n. Schultz)

3. Eiszeit

?

Sartan (?)

Syrjanka

2. Eiszeit

1. Eiszeit

Maximale

Alteste

?

?

Nordostasiatische Küsten

Japan

(n. DAMpserow \& Elisejew)

Yari

?

Komoro

?

?

Taimyrian

?

Tschuktschan

?

? 